\title{
Detection of metal ions using fluorescent gold nanoclusters
}

\author{
M. Vogel ${ }^{1,2}$, S. Matys ${ }^{1,2}$, K. Pollmann ${ }^{1}$, J. Raff ${ }^{1,2}$, \\ 1) Helmholtz-Zentrum Dresden-Rossendorf, Helmholtz Institute Freiberg for Resource Technology, \\ 09599 Freiberg, Germany \\ 2) Helmholtz-Zentrum Dresden-Rossendorf, Institute of Resource Ecology, 01328 Dresden, Germany \\ Contact: s.matys@hzdr.de
}

\begin{abstract}
S-layer proteins appear to be suitable for wide variety of different technical applications due to their distinctive physico-chemical properties and their multifunctionality. Since several years the focus has been placed especially on their potential use for biosensor applications. There are many approaches under investigation to develop sensors that are highly specific and sensitive as well as robust, reliable and not expensive. Optical methods currently appear an attractive solution. Colloidal gold nanoparticle suspensions as sensory active systems, for instance, have been the subject of intensive investigations for many years. An additional promising approach is the use of proteins as template structures for the production of highly fluorescent, size-controlled gold nanoclusters (Au-NC). These gold nanoclusters can be synthesized directly at the protein by a simple chemical reaction. We present current investigations on different kind of proteins such as bovine serum albumin, calmodulin, and S-layer protein. In combination with the known S-layer or calmodulin mediated selective and specific binding of ionic analytes, e.g. rare earth elements as surrogates/analogues for intrinsic protein bound $\mathrm{Ca}^{2+}$, a subsequent analyte-induced change in the fluorescence intensity of the gold nanoclusters might be used as sensory system for the detection of such strategic relevant elements.
\end{abstract}

Keywords S-layer proteins, BSA, gold nanoclusters, bio-sensing, fluorescence

\section{Fluorescent gold nanoclusters on bovine serum albumin}

Part of the joint project BioNEWS was the development of a new sensor concept for the detection of strategically relevant value elements from aqueous streams. For this purpose different approaches were pursued. In addition to the strategy of using sensory active living cells, the use of highly fluorescent and long-term stable gold clusters constitutes a conceivable and promising alternative. We could successfully verify a biological ("green") method of producing fluorescent gold nanoclusters using bovine serum albumin as a template first described by Xie et al. [1] and to transfer this method to other functional proteins such as calmodulin and S-layer proteins. Many proteins have a certain intrinsic reducing potential that can be used to bind ions to produce metallic or ceramic nanoparticles with particular physico-chemical properties under suitable conditions. In a one-pot reaction, fluorescent gold nanoclusters were generated on the proteinaceous support [1]. Depending on the initial $\mathrm{pH}$ different sized nanoclusters can be produced, showing different excitation and emission wavelength ranges (see Fig. 1). Blue fluorescent gold clusters are attributed to

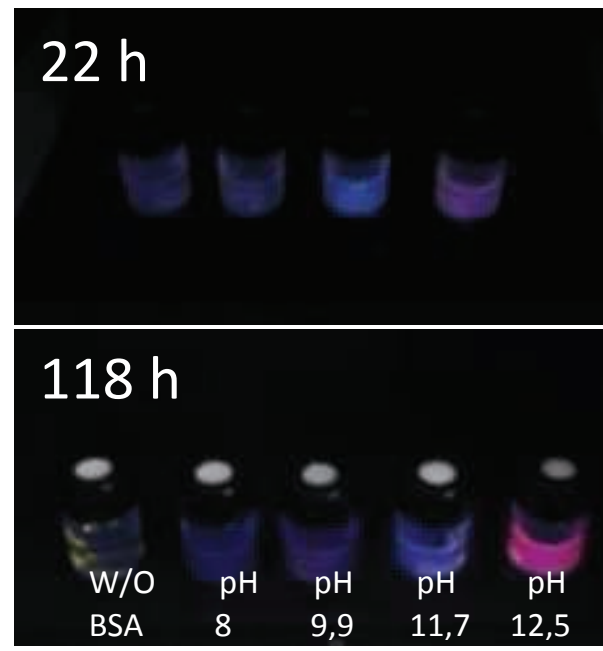

Fig. 1: BSA-AuNC suspensions with different initial $\mathrm{pH}$ under UV irradiation 22 and 118 hours after formation.

clusters of eight atoms and red to 25 atoms in size [2]. Suspensions with red-fluorescing gold clusters are stable for a period of more than 
one year and are thus ideal for the generation of long-term stable sol-gel-based sensory layers (Fig. 2).

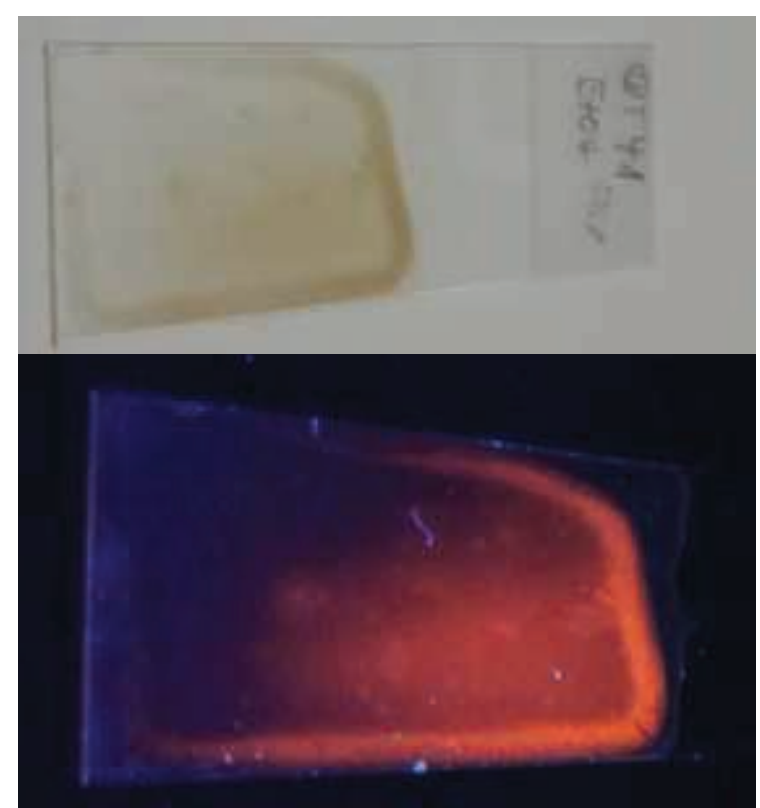

Fig. 2: BSA-AuNC suspension immobilized in TEOS sol-gel layers.

By contrast, suspensions of lower initial $\mathrm{pH}$ are not stable. Here, the smaller gold clusters tend
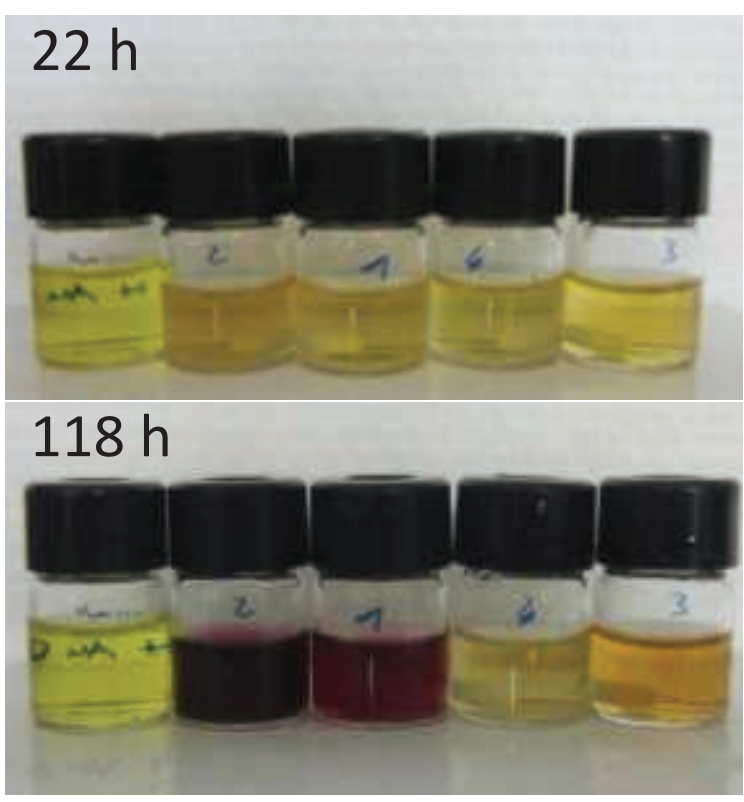

Fig. 3: Stability of BSA-AuNC suspensions with different initial $\mathrm{pH}$ values.

to agglomerate, as evidenced by ruby-red staining with the naked eye and in the UVvisible spectrum by the plasmon peak at 540 nm (Fig. 3 and 4).

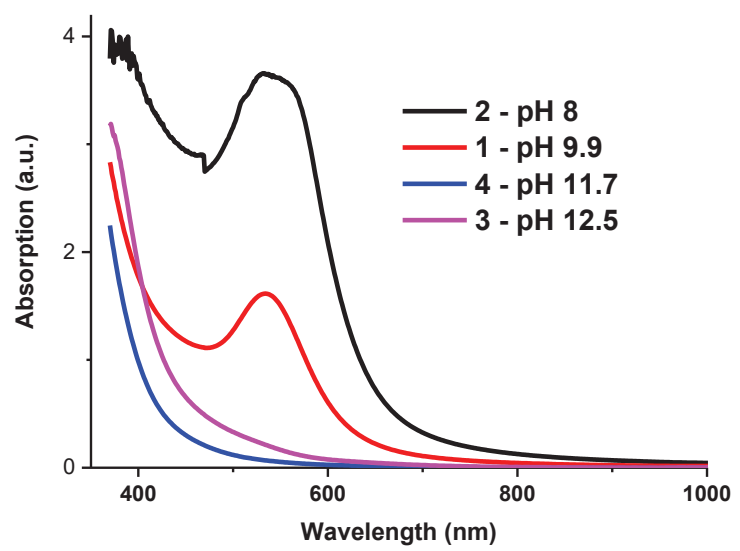

Fig. 4: UVIVIS spectra of BSA-AuNC suspensions with different initial $\mathrm{pH}$ values after $118 \mathrm{~h}$ incubation time at $37^{\circ} \mathrm{C}$.

These particles having a plasmon peak are no longer fluorescent.

\section{Sensory activity}

The sensory activity of the stable red fluorescent suspensions was tested on different analytes (Fig. 5).
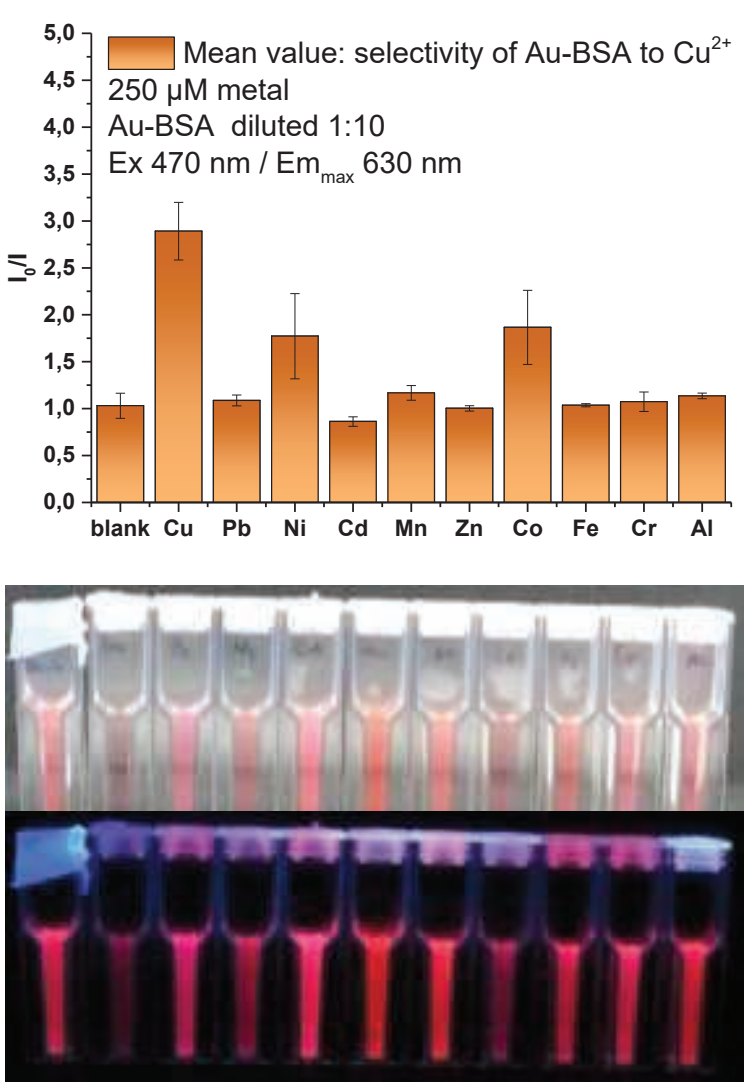

Fig. 5: Sensing of different metal ions in solution with BSA-AuNC suspensions. 
It was possible to detect a selectivity for copper (II) on the basis of a greatly reduced fluorescence intensity [3]. The decrease in the presence of cobalt and nickel was significantly weaker. For $\mathrm{Cr}, \mathrm{Al}, \mathrm{Zn}, \mathrm{Pb}$ or $\mathrm{Cd}$ little or no change in fluorescence intensity was observed. The relative fluorescence intensity was decreased as the temperature rose to $70^{\circ} \mathrm{C}$ as shown in Fig. 6, but this effect was reversible. Even after elevated temperature of $70^{\circ} \mathrm{C}$ for 24 hours the sensitivity to copper remains unaltered (Fig. 7).

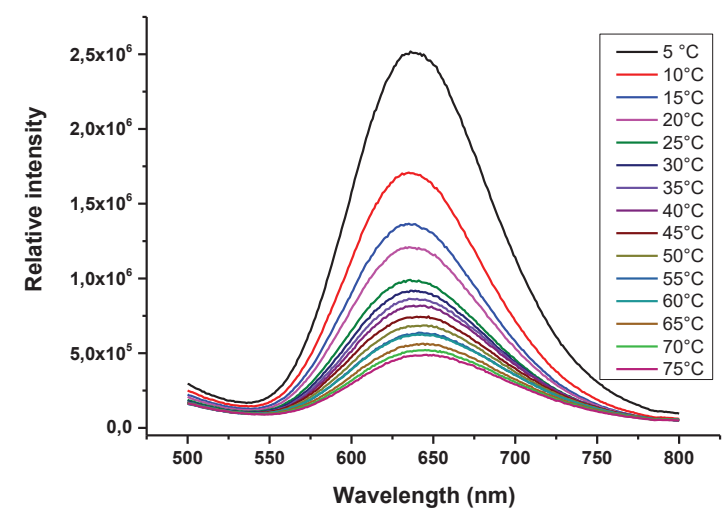

Fig. 6: Relative fluorescence intensity of $B S A-A u N C$ in dependence on the temperature
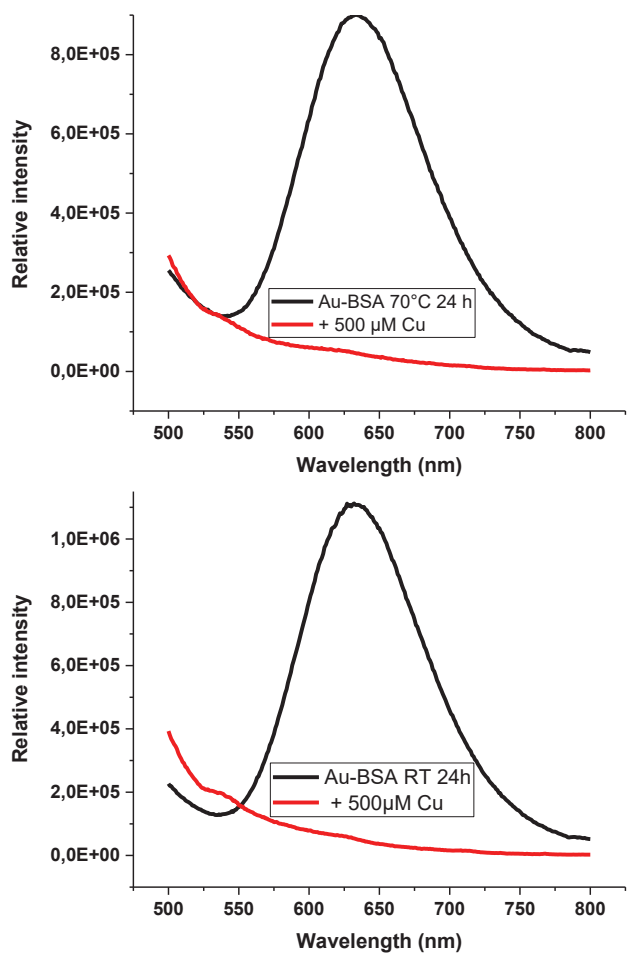

Fig. 7: Fluorescence quenching by copper ions at ambient temperature (bottom) and after $70^{\circ} \mathrm{C}$ for 24 hours (top).
The preparation of the fluorescent suspensions and the fluorescence performance after heat treatment were well reproducible. The deviations between the individual batches amounted to a maximum of $10 \%$ (Fig. 8).

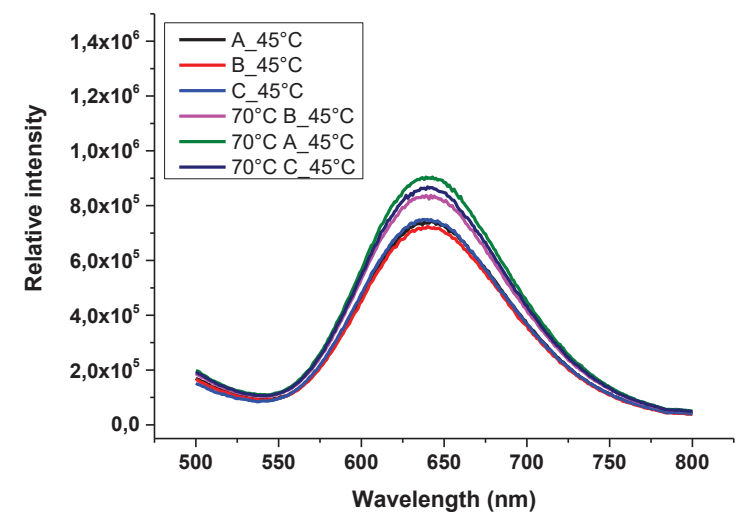

Fig. 8: Fluorescence performance of different BSA-AuNC batches after heat treatment.

\section{Fluorescent gold nanoclusters on calcium binding proteins}

In principle, the production method can be transferred to other proteins. It was possible to generate fluorescent gold clusters on two calcium-binding proteins (calmodulin and Slayer protein of Lysinibacillus sphaericus JGB53, Fig. 9 and 10).

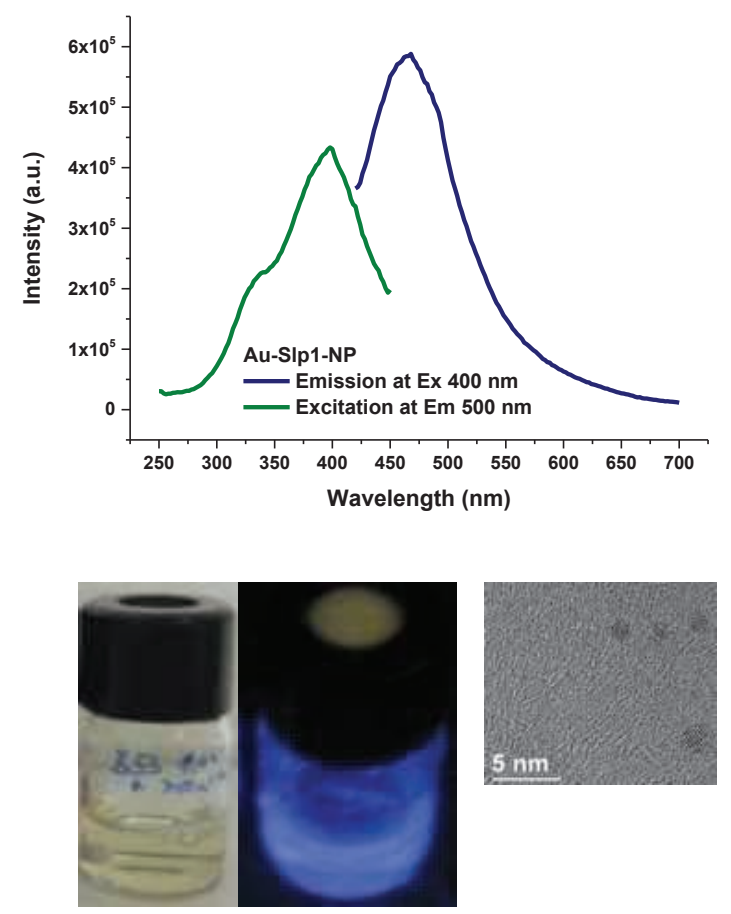

Fig. 9: Top: Excitation and emission maxima of blue fluorescent S-layer protein SIp1-AuNC, bottom left: daylight and 
UV irradiated S-layer-protein-AuNC, right: TEM image of as-synthesized metallic gold clusters on S-layer support.

Emission maxima in the blue range could be detected for both proteins, which suggest that $\mathrm{Au}_{8}-\mathrm{NC}$ were formed.

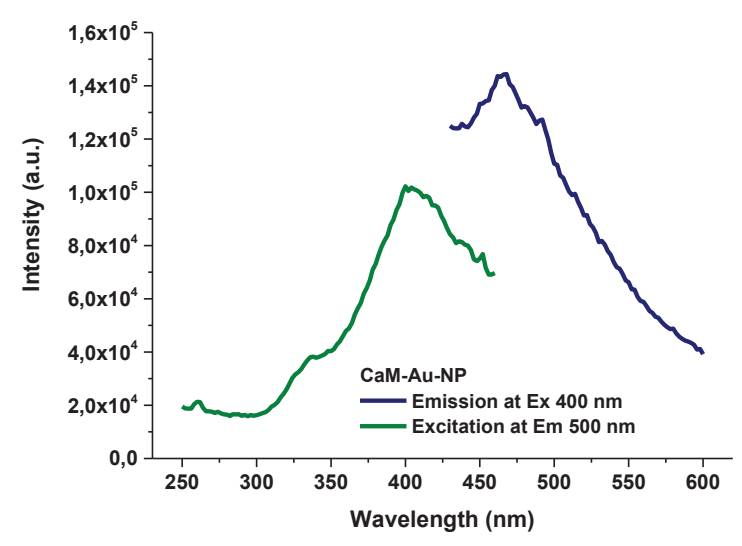

Fig. 10: Excitation and emission maxima of blue fluorescent gold nanoclusters on calmodulin.

Unfortunately, the built nanoclusters were not stable over time. It is known from the literature that the shape, size and stability of the gold clusters formed depend on the size and conformation of the protein, the presence of thiol groups and the amount of available metal precursor [4]. Neither the used S-layer protein nor calmodulin contain the sulfur-containing amino acid cysteine. The underlying mechanism of the formation of fluorescent gold nanoclusters on calcium binding proteins like S-layer proteins or calmodulin is still not known and must be different from the sulfur supported cluster formation by thiol containing proteinaceous structures. Under these conditions, the lack of stability of the fluorescent suspensions over time is not surprising. Thus, next promising steps are the modification of Ca-binding proteins with sulfhydryl groups and the use of cysteine containing S-layer proteins.

\section{Conclusions}

Long-term stable sensory active sol-gel-layers can be fabricated using highly fluorescent gold nanoclusters which were as-synthesized on bovine serum albumin. Two "magical" stable cluster sizes of eight and 25 gold atoms could be generated but only the bigger, red fluorescent, clusters were stable for more than one year. In principle, the protocol for the cluster generation can be transferred to other proteins like S-layers or calmodulin. The mechanism of cluster formation and stabilization must be different from the sulfur supported route and has to be investigated in detail.

\section{References}

[1] J. Xie, Y. Zheng, J.Y. Ying, JACS

Communications, 131, 888-889 (2009); doi: 10.1021/ja806804u

[2] X. Le Guével, B. Hötzer, G. Jung, K. Hollemeyer, V. Trouillet, M. Schneider, The Journal of Physical Chemistry C, 115, 10955-10963 (2011); doi: 10.1021/jp111820b

[3] C. V. Durgadas, C. P. Sharma, K. Sreenivasan, Analyst, 136, 933-940 (2011), doi: 10.1039/c0an00424c

[4] S. Volden, S. M. Lystvet, Ø. Halskau, W. R. Glomm, RCS Advances, 2, 11704-11711 (2012); doi: 10.1039/c2ra21931j

\section{Acknowledgement}

The project is funded by the Initiative "Unternehmen Region" of the Federal Ministry of Education and Research (BMBF) by grant number 03WKCL03F. 